\title{
The SFG (Solid, Fragmented, Granular) Index for Bacterial Morphology*
}

\author{
D. S. RIDLEY \\ Hospital for Tropical Diseases, London, N.W.1
}

The morphological index (MI) has become almost universally adopted as an indication of the viability of leprosy bacilli. This is unquestionably the correct procedure in drug trials and in many forms of research, but the determination of the MI is unnecessarily time-consuming for the routine management of patients, and also demands a high level of skill and experience on the part of the technician if reproducible and meaningful results are to be achieved.

The reason why the granularity index (Ridley, 1960) has not become more popular is probably that its simplicity is not at first sight appreciated. Dr. Tin Shwe has pointed out to me that it is also unnecessarily difficult to explain and comprehend because the values are the reverse of those of the MI. To obviate this small obstacle, it is suggested that the index values should be inverted, so that 10 represents all solid bacilli and 0 all granular bacilli. Accordingly, the index is redescribed here in inverted form, with a new name to avoid confusion. This index has been in regular routine use at this hospital for 12 years, and the technicians who have been taught its use have without exception preferred it to the morphological index. The SFG index cannot be directly equated with the MI, but it has been found entirely adequate for its purpose by clinicians.

(As a matter of historic interest, it may be mentioned that both Lowe and Davey, working in the Leprosy Research Unit, Uzuakoli (in the former Eastern Nigeria), regularly employed a similar classification of the morphology of bacilli present in routine smears.)

The SFG index. Bacilli are conveniently divided into 3 classes: (1) "solid" (S), i.e. solid-staining unbroken rods; (2) "fragmented" (F), i.e. bacilli in which the acid-fast substance is interrupted at one or more points, but at least one fragment displays an elongated form; also single very short rods; and (3) "granular" (G), i.e. round granules either in line or in clumps. It is difficult to estimate the exact percentage of bacilli in each class, but their approximate ratios can be estimated almost at a glance.

A value is assigned to the bacilli of each class in a smear: 2 if they appear numerous (over $20 \%$ of all bacilli); 1 if few (1-20\%) or 0 (if less than $1 \%$ ). Thus the relative proportion of bacilli in the 3 classes SFG (in this order) are represented by one of the permutations of 2-1-0. These combinations have been placed in order of descending granularity from 2-0-0 (all solid) to 0-0-2 (all

* Received for publication 18 February, 1971. 


\begin{tabular}{lc}
\hline \multicolumn{1}{c}{ SFG value } & SFG index \\
\hline $2-0-0$ & 10 \\
$2-1-0$ & 9 \\
$2-2-0$ & 8 \\
$2-1-1(=1-2-0)$ & 7 \\
$2-2-1$ & 6 \\
$1-2-1(=2-2-2)$ & 5 \\
$1-2-2$ & 4 \\
$1-1-2(=0-2-1)$ & 3 \\
$0-2-2$ & 2 \\
$0-1-2$ & 1 \\
$0-0-2$ & 0 \\
\hline
\end{tabular}

(The values 2-1-2, 2-0-2 and 0-2-0 are very seldom found. All give an index of 5.)

granular) to give an index, as shown in the accompanying column. The order is not obvious. Based on the ratios of solid to granular bacilli it was determined by plotting the various SFG values in the form of crude distribution curves on squared paper and counting the squares on either side of the " $F$ " values. If several smears are available the mean index is taken.

\section{References}

Ridley, D. S. (1960). The bacteriologic study of erythema nodosum leprosum. Int. J. Lepr. 28, 254. 